\title{
Studies on Fundamental Interaction Parameters for Stainless Steel and Titanium Biomaterials Using Flattened and Un-Flattened Megavoltage X-Ray Beams
}

\author{
Tamilarasan Rajamanickam ${ }^{1,2}$, Sivakumar Muthu', Perumal Murugan', \\ Muddappa Pathikonda ${ }^{1}$, Krishnamoorthy Senthilnathan ${ }^{2}$, Narayanasamy Arunai \\ Nambi Raj ${ }^{3}$, Padmanabhan Ramesh Babu ${ }^{2 *}$
}

\begin{abstract}
Purpose: This work presents the measure of fundamental interaction parameters like mass attenuation coefficient $(\mu / \rho)$, mean energy, total atomic $\left(\sigma_{\mathrm{a}}\right)$ and electronic $\left(\sigma_{\mathrm{e}}\right)$ cross section, effective atomic number $\left(\mathrm{Z}_{\text {eff }}\right)$, electron density $\left(\mathrm{N}_{\mathrm{el}}\right)$ and mean free path (mfp) using FF and UF megavoltage $\mathrm{x}$-ray beam for high $\mathrm{Z}$ implants. Methods: Narrow beam geometry is used to find out mass attenuation coefficient $(\mu / \rho)$ (MAC) which is then used to calculate mean energy (using NIST data), total atomic $\left(\sigma_{\mathrm{a}}\right)$ and electronic cross section $\left(\sigma_{\mathrm{e}}\right)$ for different energies. The effective atomic number $\left(\mathrm{Z}_{\text {eff }}\right)$, Electron density $\left(\mathrm{N}_{\mathrm{el}}\right)$, mean free path $(\mathrm{mfp})$ for both flattened and unflattened x-ray beams for high $\mathrm{Z}$ material stainless steel (SS316) and titanium alloy (Grade 5) are studied. Results: The mean energies calculated from $\mathrm{N}_{\text {IST }}$ data against mass attenuation coefficient were in good agreement with Monte Carlo value. It shows that spectral weighted effective atomic number is independent of megavoltage energies in the Compton region. Effective electron density calculated using Zeff and MAC method is lesser compared to direct method for both high Z materials. The mean free path (mfp) is higher along the central axis than off-axis for flattened beam in comparison to unflattened beam for both of the high $\mathrm{Z}$ materials studied because of the variation in energy spectrum for both FF and UF x-ray beams. Conclusion: This study elaborated the fundamental interaction parameters of different energies of flattened and unflattened $x$-ray beam interactions with high Z materials such as Stainless Steel (SS316) and Titanium (Grade5) relevant in a clinical scenario.
\end{abstract}

Keywords: Flattened (FF) and unflattened (UF) x-ray beams- mass attenuation coefficient- electron density

Asian Pac J Cancer Prev, 20 (8), 2485-2491

\section{Introduction}

With the introduction of unflattened x-ray beams in cancer treatment, the energy spectrum changes drastically with the removal of flattening filter from $\mathrm{x}$-ray beam path (Cashmore et al., 2008; Goerg et al., 2011; Kargl et al., 2009). Therefore, the mean energy at isocenter and off-axis varies considerably between unflattened and flattened beam. In radiation therapy, incident of composite materials (Implant material) like hip prosthesis, vertebral reconstruction, and tooth implants are high. Those are alloys with a high atomic number that are hard-wearing and bio-compatible. The conflict between high $\mathrm{Z}$ implant material and flattened, unflattened x-ray beams are often observed; this conflict degrades the dose distribution (Chester et al., 2003; Papinakolaou et al., 2004) as these implanted high $\mathrm{Z}$ materials have different interaction properties than other low $\mathrm{Z}$ materials such as tissue, lungs, cartilages and bone. Hence, it is important to address the variation in behavior of fundamental parameters when x-ray beams of different mean energies interacting with high $\mathrm{Z}$ composite materials or alloys in flattened and unflattened megavolt x-ray beams (Koc et al., 2000).

Fundamental parameters like mass attenuation coefficient (MAC), mean energy, total atomic $\left(\sigma_{\mathrm{a}}\right)$ and electronic $\left(\sigma_{\mathrm{e}}\right)$ cross-sections, effective atomic number $\left(\mathrm{Z}_{\text {eff }}\right)$, effective electron density $\left(\mathrm{N}_{\mathrm{e}}\right)$, and photon mean free path $(\mathrm{mfp})$ are used to study the interaction of photon with matter. MAC is a measure of probability of interaction that occurs between incident photons and unit per mass area of matter. MAC is widely used in the calculation of photon penetration and energy deposition in biological shielding and other dosimetric materials (Kiran Kumar et al., 1997).

For a given composite material absorber (High Z implants), an interaction cross-section $(\sigma)$ varies as a function of wavelength or energy of flattened and 
unflattened megavoltage $\mathrm{x}$-ray beams. The cross-section is not the probability of interaction, but the probability of interaction is proportional to the cross-section. A slower projectile (low energy) spends more time in the proximity of the target; hence, it has a higher probability of interaction. The cross-section, expressed in terms of barns $\left(10^{-24} \mathrm{~cm}\right)$, is the effective "area of interaction" per absorber at energy (E) or wavelength $(\lambda)$ of flattened and unflattened x-ray beams (Hine, 1952). The effective atomic number and electron density are convenient parameters used for better understanding of the interaction of radiation with the target material and accurately estimate the absorbed dose. As effective atomic number and electron densities are dependent on energy of incident $\mathrm{x}$-ray beams, it is important to study the impact of varied energy spectrum of flattened and unflattened $\mathrm{x}$-ray beams on these parameters in megavoltage region (Compton region). As energy spectrum of flattened and unflattened $\mathrm{x}$-ray beam varies each other and varies in its field itself, the mean free path varies in high $\mathrm{Z}$ materials too where this may occur in the treatment field.

The energy absorption in a given medium can be calculated if certain constants, such as effective atomic number and electron density of the medium, are known. Since these two parameters are useful in many technological applications, several investigators (Kaginelli et al., 2009; Manohara et al., 2008) have made extensive studies on effective atomic numbers in a variety of composite materials like alloys, polymers, compounds, mixtures, thermoluminescent dosimetric compounds, semiconductors and superconductors using kilovoltage and very few with megavoltage $\mathrm{x}$-ray beams with high $\mathrm{Z}$ implant material.

In this work, we studied the interaction parameters of bio-compatible medical grade high $\mathrm{Z}$ stainless steel (SS-316) and titanium alloy (Grade 5) implants using flattened and unflattened megavoltage $x$-ray beams in the presence of high $\mathrm{Z}$ implant materials while treating cancer patient in radiotherapy.

\section{Materials and Materials}

In this work, a TrueBeam 2.0 (Varian Medical System Inc., Palo Alto, CA, USA), which is capable of delivering flattened and unflattened $\mathrm{x}$-ray beams of $6 \mathrm{MV}(6 \mathrm{FF}), 10$ MV (10FF), $15 \mathrm{MV}$ (15FF) and 6 MVFFF (6UF), and 10 MVFFF (10UF) were used in conjunction with high Z stainless steel (SS316) and titanium alloy (Grade 5) as they are of austenitic grades (nonmagnetic). The effective atomic number of stainless steel (SS316) and titanium (Grade 5) are 29.23 and 22.15 respectively and the average mass numbers (A) are $56.32 \mathrm{u}$ and $46.66 \mathrm{u}$ respectively. The composition of stainless steel (SS316) and titanium (Grade5) materials is listed in table 1 (Weisner ME et al., 2013). The stainless steel (SS316) and titanium (Grade 5) used in this study were of dimension $3 \times 3 \times 2.5 \mathrm{~cm}^{3}$ with densities of $8.1 \mathrm{~g} / \mathrm{cm}^{3}$ and $4.3 \mathrm{~g} / \mathrm{cm}^{3}$ respectively.

The experimental setup for measuring the MAC was carefully done to have "good geometry" with narrow beam conditions. The measurements were carried out in air using $\mathrm{CC} 13$ ionization chamber with appropriate build-up cap to achieve electronic equilibrium. The chamber was positioned at isocenter $100 \mathrm{~cm}$ from target (x-ray source), and the high Z shielding materials (SS316 and Ti Grade 5) were placed in air $10 \mathrm{~cm}$ above the detection system in between the target and the detector. The distance between detector and high $\mathrm{Z}$ materials were maintained to avoid any scattered electrons reaching the detector as this may lead to underestimation of the dose (TRS No. 398, 2000). The measurements were carried out both along the central axis $(0,0,0)$ and off-axis $(15,0,0)$ of about $15 \mathrm{~cm}$ from CAX to determine the variation in mean energy at off-axis by changing chamber position to $15 \mathrm{~cm}$ off axis. We assumed that measuring the MAC at off-axis can be mirrored in opposite side as variation in energy spectrum in off-axis is same from isocenter $(0,0$, 0 ) coordinates for flattened and unflattened $x$-ray beams. The central and off-axis measurements were carried out for the field size of $3 \times 3 \mathrm{~cm}^{2}$.

\section{Theoretical Methods}

The effective mass attenuation coefficient of the given compounds (High Z alloys: SS316 and Ti Grade 5) were obtained from the well-known Beer-Lambert attenuation law. These co-efficient were then used for calculation of mean energy, total atomic and electronic cross-section, effective atomic number, electron density, and mean free path.

\section{Mass attenuation co-efficient $(\mu / \rho)$}

The total MAC $(\mu / \rho)$ compound for any mixture of element characterizes the measure of the probability of interaction of incident photons within the thickness $\left(\mathrm{g} / \mathrm{cm}^{3}\right)$ of the target material. It is given by,

$$
\frac{\mu}{\rho}=\frac{1}{x} \ln \left(\frac{I_{\mathrm{o}}}{I}\right)
$$

Here, $\mu / \rho$ is MAC, $I_{0}$ and I are the intensity of beam with and without attenuation material respectively, and $\mathrm{x}$ is the thickness of attenuation material such that $x=\rho t$ ( $\rho$ is the density of compound, $t$ is the thickness of compound). If the attenuation material is a composite of different elements, then the resultant will be multiplied over weightage $w_{i}$ of each element in the compound (Kerur et al., 1993). The effective MAC was derived as follows:

$$
\left(\frac{\mu}{\rho}\right) \text { compound }=\sum_{i} w_{i}\left(\frac{\mu}{\rho}\right)
$$

\section{Total Atomic Cross-Section $(\sigma)$}

The total atomic cross-section $\left(\sigma_{\mathrm{a}}\right)$ is given by the following formula

$$
\sigma_{a}=\frac{N_{i}}{N_{A}}\left(\frac{\mu}{\rho}\right) \text { Compound }
$$

where $N_{i}$ is the atomic mass of the material and $N_{A}$ is the Avogadro's number

The total cross-section can be written as the sum over contributions from the principle photon interactions,

$$
\sigma_{\text {tot }}=\sigma_{\text {pe }}+\sigma_{\text {coh }}+\sigma_{\text {incoh }}+\sigma_{\text {pair }}+\sigma_{\text {trip }}+\sigma_{\text {ph.n. }}
$$


where $\sigma_{p e}$ is the atomic photoelectric effect cross-section; $\sigma_{\text {coh }}$ and $\sigma_{\text {incoh }}$ are the coherent (Rayleigh) and the incoherent (Compton) scattering cross-sections, respectively; $\sigma_{\text {pair }}$ and $\sigma_{\text {trip }}$ are the cross-sections for electron-positron production in the fields of the nucleus and of the atomic electrons, respectively; and $\sigma_{\text {ph.n. }}$ is the photonuclear cross-section.

\section{Total Electronic Cross-Section ( $\sigma e$ )}

The total electronic cross-section $\left(\sigma_{\mathrm{e}}\right)$ is given by the following formula:

$$
\sigma_{e}=\frac{1}{N_{A}} \sum_{i} \frac{f_{i} N_{i}}{Z_{i}}\left(\frac{\mu}{\rho}\right) \text { Compound }
$$

where $f_{i}$ denotes the fractional abundance of the element $\mathrm{i}$ with respect to the number of atoms such that $f_{1}+f_{2}+\ldots .+f_{i}=1$ and $\mathrm{Z}_{\mathrm{i}}$ is the atomic number of ith element.

\section{Effective Atomic Number $\left(Z_{\text {eff }}\right)$ \\ (a) Power-law method}

The power law method dates back to 1930s when the photon sources were restricted to low energy $\mathrm{X}$ ray beam. The following proposed formula is used to calculate effective atomic number (Khan, 1994).

$$
Z_{\text {eff }}=\sqrt[2.94]{f_{1} \times\left(Z_{1}\right)^{2.94}+f_{2} \times\left(Z_{2}\right)^{2.94}+f_{3} \times\left(Z_{3}\right)^{2.94}+\cdots}
$$

where $f_{1}$ is the fractional number of the electrons associated with each element, and $Z_{n}$ is atomic number of the respective element.

\section{(b) Direct method}

The effective atomic number of the material can be calculated by determining the total attenuation cross-section of atomic $\left(\sigma_{\mathrm{a}}\right)$ and electronic $\left(\sigma_{\mathrm{e}}\right)$ as shown in the following equation (Graham et al., 2012),

$$
Z_{e f f}=\frac{\sigma_{a}}{\sigma_{e}}
$$

\section{(c) Auto- $Z_{\text {eff }}$ method}

Auto- $Z_{\text {eff }}$ method is user-friendly software version 1.7 (Taylor et al., 2012) that uses visual basic for calculating the average atomic number and spectral weighted mean atomic numbers for elements or composites. The Auto- $Z_{\text {eff }}$ method follows the uncertain power-law approach. Here, the effective atomic number is calculated through the exploitation of the smooth correlation between atomic cross-section and atomic number. The tabulation of cross-section was constructed covering the atomic number $Z=1-100$ for photon energies ranging between 10 $\mathrm{KeV}$ and $1 \mathrm{GeV}$, and the cross-section of composite media was calculated with linear additivity. The cross-sectional values were constructed with the cross-section matrix as a function of $Z$, and the effective atomic number was obtained by the interpolation of $Z$ values between adjacent cross-section values.

\section{(d) XMuDat method}

The XMuDat program can produce a single value of effective atomic number for compounds. This program uses the following formula for calculating the effective atomic number (Nowotny et al., 1998).

$$
Z_{e f f}(\text { XMuDat })=\left[\sum_{i} \alpha_{i} Z i^{m-1}\right]^{1 /(m-1)}
$$

where $\alpha_{i}$ is the fractional number of the electrons of the ith element, and $\mathrm{m}$ is a constant between 3 and 5. It is preferred that $\mathrm{m}$ is set to 3.6 for material with $\mathrm{Z}_{\text {eff }}<6$ and 4.1 for material with $\mathrm{Z}_{\mathrm{eff}}>6$.

\section{Electron Density}

The probability of Compton scattering occurring per unit mass is inversely proportional to the energy of the incident photon and directly proportional to electron density. In megavoltage beam, the most predominant interaction is Compton Effect which depends on electron density rather than atomic number. The number of electrons is same as the number of protons and is given by atomic number $(Z)$. The electron density is the number of electrons per unit mass and is given by the equation below:

$$
\text { electron density }=N_{A} X \frac{Z}{A}
$$

If we assume that most of the elements in tissue have approximately an equal number of protons and neutrons in the atomic nucleus, then the value of $\mathrm{Z} / \mathrm{A}$ is 0.5 . The exception to this rule is hydrogen, which contains no neutron in its nucleus and so, the value of $\mathrm{Z} / \mathrm{A}$ is 1 . Thus, hydrogen contains $6 \times 10^{23}$ electrons per gram. All other substances contain approximately half of this value (between $2.5 \times 10^{23}$ and $3.5 \times 10^{23}$ electrons per gram). Lower values were observed for the heavier elements that have larger neutron-proton ratio (Shivaramu, 2002). The experimental way of calculating effective electron number or electron density $\mathrm{N}_{\mathrm{el}}$ (number of electron per unit mass) can be written as,

$$
\begin{aligned}
N_{e l} & =\frac{N_{A}}{A} Z_{\text {eff }} \sum n i \\
N_{e l} & =\frac{x\left(\frac{\mu}{\rho}\right)_{\text {Compound }}}{\sigma_{e}}
\end{aligned}
$$

Where, the $Z_{\text {eff }}$ and $(\mu / \rho)_{\text {Compound }}$ are experimentally calculated effective atomic number and $\mathrm{MAC}$, and $\mathrm{A}$ is average atomic mass (AAM) of compounds.

\section{Mean Free Path}

The average distance between two successive interactions called the photon mean free path is given by

$$
m f p=\frac{1}{\mu}
$$

Where, $\mu$ is the linear attenuation coefficient. In linear accelerator-based radiotherapy, the calculation of mean free path is much more complicated owing to the fact that photons are not mono-energetic and their energy is defined by an energy spectrum. The mean free path value can be incorporated with a mean energy of energy spectrum. As photons move through the target material, they are attenuated with probabilities depending on their energy. As a result, their distribution changes in a process called spectrum hardening. Due to spectrum hardening, the mean 
free path of the $\mathrm{x}$ - ray spectrum changes with distance.

\section{Results}

\section{Mass Attenuation Coefficient $(\mu / \rho)$}

The MAC $(\mu / \rho)_{\text {compound }}$ calculated $( \pm 0.1 \%)$ for all energies $6 \mathrm{FF}, 10 \mathrm{FF}, 15 \mathrm{FF}, 6 \mathrm{UF}$ and $10 \mathrm{UF}$ with narrow beam geometry $( \pm 1 \mathrm{~mm})$ for stainless steel (SS316) and titanium (Grade5) using formula 1 and it shows that as energy increases, the MAC decreases in both flattened and unflattened $\mathrm{x}$-ray beams. The MAC increases with unflattened beam than with flattened beam of same energy as the mean energy is lower in unflattened beam due to beam softening (removing flattening filter from $\mathrm{x}$-ray beam path). The variation in MAC was also studied for off-axis in order to identify the magnitude of variation in mean energy from central axis (Zefkili et al., 1994). The results show that the variation in MAC is larger from the central axis to off-axis for flattened beam than compared to unflattened beam (Table 2).

\section{Mean Energy}

The calculated MAC $(\mu / \rho)_{\text {compound }}$ is counter checked with total MAC with coherent scattering values against $\mathrm{X}$-ray photon energies created for stainless steel and titanium with exact composition as mentioned in Table 1 using NIST-XCOM software version 1.7 (Berger at al., 1987). The energy was interpolated against experimental MAC $(\mu / \rho)_{\text {compound }}$.

The energy interpolated from NIST-XCOM for each composition was taken and compared with the mean energy calculated from Monte Carlo value (Dalaryd et al., 2010). The results showed that the mean energies calculated from MAC and Mote Carlo was in good agreement with each other (Table 3). Although the NIST- XCOM table gives findings for mono-energetic beam, the MAC for spectral beam was equated to determine the resultant mean energy. This experiment can be useful in finding the variation of energy spectrum throughout the field opening while commissioning the flattened and unflattened x-ray beams using known composition of attenuating material.

\section{Total Atomic Cross-Section $\left(\sigma_{d}\right)$}

The value of $\operatorname{MAC}(\mu / \rho)$ compound (form Table 2) were used to obtain the total atomic cross-section $\left(\sigma_{\mathrm{a}}\right)( \pm 0.1 \%)$ from formula 3 for compound material (alloys) of stainless steel (SS316) and titanium (Grade 5). The total atomic cross-section $\left(\sigma_{\mathrm{a}}\right)$ decreases with the increase in energy as shown in Table 4 for both flattened and unflattened $x$-ray beam. Also, the cross-section increases in unflattened beam than in flattened $\mathrm{x}$-ray beam due to the decrease in
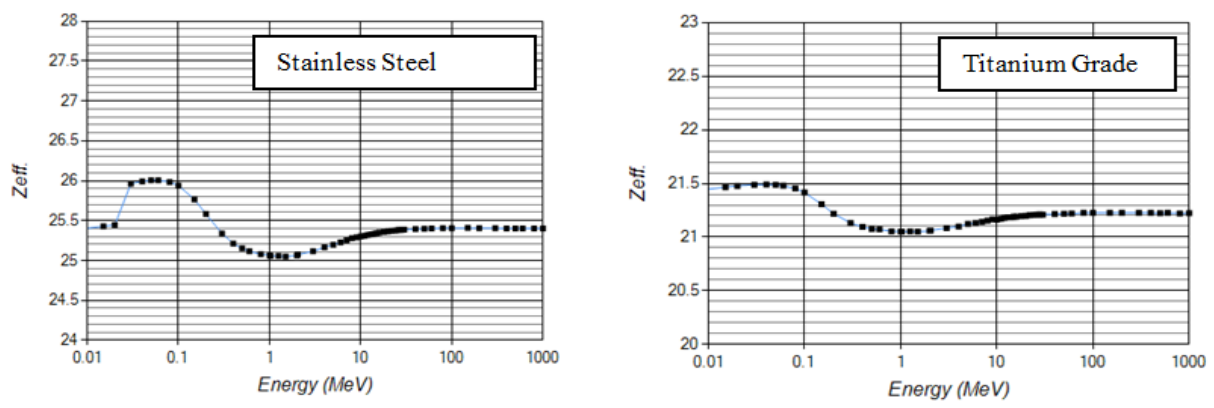

Figure 1. Energy Dependency of $\mathrm{Z}_{\text {eff }}$ for Stainless Steel and Titanium

Table 1. Physical and Chemical Properties of Stainless Steel (SS316) and Titanium Alloy (Grade 5)

\begin{tabular}{llcccc}
\hline Material & Chemical Composition & \% of Composition & Z & A & $\rho\left(\mathrm{g} / \mathrm{cm}^{3}\right)$ \\
\hline Stainless Steel (SS316) & & & & \\
& Iron (Fe) & 65.35 & 26 & 56 & 7.9 \\
& Chromium (Cr) & 17.00 & 24 & 52 & 7.2 \\
& Nickel (Ni) & 12.00 & 28 & 59 & 8.9 \\
& Molybdenum (Mo) & 2.50 & 42 & 96 & 10.28 \\
& Manganese (Mn) & 2.00 & 25 & 55 & 7.43 \\
& Silicon (Si) & 1.00 & 14 & 28 & 2.33 \\
& Carbon (C) & 0.08 & 6 & 12 & 2.25 \\
& Phosphorus (P) & 0.05 & 15 & 31 & 1.83 \\
& Sulfur (S) & 0.03 & 16 & 32 & 2 \\
& & & & \\
& Titanium (Ti) & 89.55 & 22 & 48 & 4.5 \\
& Aluminum (Al) & 6.00 & 13 & 27 & 2.7 \\
& Vanadium (V) & 4.00 & 23 & 50.9 & 6.1 \\
& Iron (Fe) & 0.25 & 26 & 56 & 7.9 \\
& Oxygen (O) & 0.20 & 8 & 16 & 1.43 \\
\hline
\end{tabular}


Table 2. Measured Mass Attenuation Coefficient (MAC) for Stainless Steel and Titanium at Central and off-Axis

\begin{tabular}{lcccccc}
\hline \multirow{2}{*}{ Energy (MV) } & \multicolumn{5}{c}{ Mass Attenuation Coefficient $(\mu / \rho)_{\text {Compound }} \mathrm{cm}^{2} / \mathrm{g}$} \\
& CAX $(0 \mathrm{~cm})$ & Stainless Steel (SS) & \multicolumn{3}{c}{ Titanium (Ti) } \\
\hline $6 \mathrm{FF}$ & 0.04288 & 0.04701 & $9.60 \%$ & 0.04541 & 0.04938 & $8.70 \%$ \\
$10 \mathrm{FF}$ & 0.03536 & 0.03948 & $11.60 \%$ & 0.0350 & 0.03958 & $11.40 \%$ \\
$15 \mathrm{FF}$ & 0.03418 & 0.03781 & $10.60 \%$ & 0.03225 & 0.03575 & $10.80 \%$ \\
$6 \mathrm{UF}$ & 0.04931 & 0.05079 & $3.00 \%$ & 0.05056 & 0.05213 & $3.10 \%$ \\
$10 \mathrm{UF}$ & 0.04010 & 0.04199 & $4.70 \%$ & 0.04126 & 0.04259 & $3.20 \%$ \\
\hline
\end{tabular}

Table 3. Mean Energy by Monte Carlo and Using NIST XCOM Data (By MAC Value) for Flattened and Un-flattened X-ray Beam

\begin{tabular}{|c|c|c|c|c|c|c|}
\hline \multirow[b]{3}{*}{ Energy (MV) } & \multicolumn{4}{|c|}{ Mean Energy (MeV) } & & \\
\hline & \multicolumn{2}{|c|}{ Monte Carlo } & \multicolumn{2}{|c|}{ Stainless Steel (SS316) } & \multicolumn{2}{|c|}{ Titanium (Ti) } \\
\hline & $\mathrm{CAX}(0 \mathrm{~cm})$ & $\mathrm{OAD}(15 \mathrm{~cm})$ & $\mathrm{CAX}(0 \mathrm{~cm})$ & OAD $(15 \mathrm{~cm})$ & $\mathrm{CAX}(0 \mathrm{~cm})$ & OAD $(15 \mathrm{~cm})$ \\
\hline $6 \mathrm{FF}$ & 1.96 & 1.50 & 1.92 & 1.58 & 1.71 & 1.40 \\
\hline $10 \mathrm{FF}$ & 2.96 & 2.37 & 2.96 & 2.36 & 2.94 & 2.34 \\
\hline $15 \mathrm{FF}$ & - & - & 3.67 & 2.79 & 3.84 & 2.81 \\
\hline $6 \mathrm{UF}$ & 1.65 & 1.50 & 1.52 & 1.42 & 1.50 & 1.38 \\
\hline 10UF & 2.37 & 2.10 & 2.26 & 2.00 & 2.23 & 1.96 \\
\hline
\end{tabular}

mean energy for unflattened x-ray beam. As mentioned in MAC, the total atomic cross-section is also affected similarly at off-axis $(15 \mathrm{~cm})$ from beam central axis and the variation is higher in flattened $\mathrm{x}$-ray beam in all energies than in unflattened $x$-ray beam. The macroscopic cross section $\left(\mathrm{cm}^{-1}\right)$ of high $\mathrm{Z}$ medium required for clinical treatment calculation algorithms can be obtained from the total atomic cross section and mass density of that particular material. Therefore, this variation in total atomic cross section due to flattened and unflattened $\mathrm{x}$-ray beams will impact the scattering process and hence the varied interaction with in the medium.

\section{Total Electronic Cross-Section $\left(\sigma_{e}\right)$}

As in the case of total atomic cross-section $\left(\sigma_{\mathrm{a}}\right)$, the total electronic cross-section is derived $( \pm 0.1 \%)$ from $\operatorname{MAC}(\mu / \rho)$ compound (Table 2 ) from formula 5.

The total electronic cross-section $\left(\sigma_{\mathrm{e}}\right)$ decreases with the increase in energy for both flattened and unflattened $\mathrm{x}$-ray beams, as observed for the total atomic cross-section $\left(\sigma_{\mathrm{a}}\right)$. As the mean energy decreases, the total electronic cross-section increases for the same flattened and

Table 4. Total Atomic Coss-Section $(\sigma)$ for Stainless Steel and Titanium for Flattened and un-Flattened $x$-Ray Beam for Central and Off-Axis

\begin{tabular}{lcccc}
\hline \multicolumn{5}{c}{ Total Atomic Cross-section $\left(\sigma_{\mathrm{a}}\right)\left(\mathrm{x} 10^{-24} \mathrm{~cm}^{2} /\right.$ atom $)$} \\
Energy & Ctainless Steel $(\mathrm{SS} 316)$ & \multicolumn{2}{c}{ Titanium $($ Grade 5) } \\
$(\mathrm{MV})$ & $(0 \mathrm{~cm})$ & $(15 \mathrm{~cm})$ & $(0 \mathrm{~cm})$ & $(15 \mathrm{~cm})$ \\
\hline $6 \mathrm{FF}$ & 4.01019 & 4.39626 & 3.52101 & 3.8284 \\
$10 \mathrm{FF}$ & 3.30709 & 3.69212 & 2.75325 & 3.06878 \\
$15 \mathrm{FF}$ & 3.19657 & 3.53634 & 2.50013 & 2.77195 \\
$6 \mathrm{UF}$ & 4.61115 & 4.75033 & 3.91993 & 4.19664 \\
$10 \mathrm{UF}$ & 3.37064 & 3.92758 & 3.19910 & 3.30242 \\
\hline
\end{tabular}

unflattened x-ray beam energies. An increase in total electronic cross-section is observed off-axis $(15 \mathrm{~cm}$ from central axis) since the mean energy decreases laterally (Table 5).

\section{Effective Atomic Number $\left(Z_{\text {eff }}\right)$}

In Compton scattering region (megavoltage region), the effective atomic number calculated by cross section, auto $\mathrm{Z}_{\text {eff }}$ and $\mathrm{XMuDat}$ methods were of the same order (Table 6). This indicates that the effective atomic number remains invariable for flattened and unflattened megavoltage $\mathrm{x}$-ray beams because of predominant Compton region.

For better understanding of $Z_{\text {eff }}$ for various interaction regions, Figure 1 denotes that how effective atomic number varies with respect to mono energetic $\mathrm{X}$-ray beam (10 KeV-1 GeV). It shows that for stainless steel (SS316) and titanium (Grade5), the effective atomic number $\left(Z_{\text {eff }}\right)$ increases in lower energies where photo electric effect is predominant; this depends on $\mathrm{Z}^{3}$ and $1 / \mathrm{E}^{3}$, hence, there is more attenuation cross-section in this energy range. The effective atomic number decreases beyond $0.1 \mathrm{MeV}$

Table 5. Total Electronic Cross-Section $(\sigma)$ for Stainless Steel and Titanium for Flattened and un-Flattened $\mathrm{x}$-Ray Beam for Central and Off-Axis

\begin{tabular}{|c|c|c|c|c|}
\hline \multicolumn{5}{|c|}{ Total Electronic Cross-section $(\sigma e)\left(\times 10^{-25} \mathrm{~cm}^{2} /\right.$ electron $)$} \\
\hline \multirow[b]{2}{*}{$\begin{array}{l}\text { Energy } \\
\text { (MV) }\end{array}$} & \multicolumn{2}{|c|}{ Stainless Steel (SS316) } & \multicolumn{2}{|c|}{ Titanium (Grade 5) } \\
\hline & $\begin{array}{l}\text { CAX } \\
(0 \mathrm{~cm})\end{array}$ & $\begin{array}{c}\text { OAD } \\
(15 \mathrm{~cm})\end{array}$ & $\begin{array}{l}\text { CAX } \\
(0 \mathrm{~cm})\end{array}$ & $\begin{array}{c}\text { OAD } \\
(15 \mathrm{~cm})\end{array}$ \\
\hline $6 \mathrm{FF}$ & 1.53294 & 1.68052 & 1.64113 & 1.7844 \\
\hline $10 \mathrm{FF}$ & 1.26417 & 1.41135 & 1.28328 & 1.43034 \\
\hline $15 \mathrm{FF}$ & 1.22193 & 1.35181 & 1.1653 & 1.29199 \\
\hline $6 \mathrm{UF}$ & 1.76266 & 1.81587 & 1.82706 & 1.95603 \\
\hline $10 \mathrm{UF}$ & 1.43372 & 1.50136 & 1.49109 & 1.53924 \\
\hline
\end{tabular}

Asian Pacific Journal of Cancer Prevention, Vol 202489 
Table 6. Effective Atomic Number $\left(Z_{\text {eff }}\right)$ Using Different Methods for All Studied Energies

\begin{tabular}{|c|c|c|c|c|c|}
\hline \multirow[t]{3}{*}{ High Z Material } & \multicolumn{5}{|c|}{ Effective Atomic Number (Zeff) } \\
\hline & \multirow[t]{2}{*}{ Power Law Method } & \multirow[t]{2}{*}{ Cross Section Method } & \multicolumn{2}{|c|}{ Auto $Z_{\text {eff }}$} & \multirow[t]{2}{*}{ XmuDat } \\
\hline & & & Spectral Weighted & Mean & \\
\hline Stainless Steel (SS316) & 29.4 & 25.7 & 25.5 & 25.2 & 26.48 \\
\hline Titanium (Grade 5) & 22.15 & 21.15 & 21.5 & 21.1 & 21.69 \\
\hline
\end{tabular}

Table 7. Effective Electron Density $\left(\mathrm{N}_{\mathrm{el}}\right)$ for Stainless Steel (SS316) and Titanium (Grade 5) for All Studied Energies

\begin{tabular}{|c|c|c|c|c|c|c|}
\hline High Z Material & $\begin{array}{l}\text { Weighted Atomic } \\
\text { Number }(\mathrm{Zi})^{*}\end{array}$ & $\begin{array}{c}\text { Avg } \\
\text { Atomic Mass }(\mathrm{A})^{*}\end{array}$ & $\begin{array}{c}\text { Eff } \\
\text { Atomic Number }\left(Z_{\text {eff }}\right) * *\end{array}$ & $\begin{array}{r}\boldsymbol{N e l}=\boldsymbol{N}_{\boldsymbol{A}} \boldsymbol{X} \frac{\boldsymbol{Z} \boldsymbol{i}}{\boldsymbol{A}} \\
\mathrm{X} 10^{23} \mathrm{e} / \mathrm{g}\end{array}$ & $\begin{array}{c}\boldsymbol{N e l}=\boldsymbol{N}_{\boldsymbol{A}} \boldsymbol{X} \frac{\boldsymbol{Z e f f}}{\boldsymbol{A}} \\
\mathrm{X} 10^{23} \mathrm{e} / \mathrm{g}\end{array}$ & $\begin{array}{c}\mathrm{Nel}=\frac{(\mu / \boldsymbol{\rho}) \operatorname{Com} \boldsymbol{p}}{\boldsymbol{\sigma} \boldsymbol{e}} \\
\mathrm{X} 10^{23} \mathrm{e} / \mathrm{g}\end{array}$ \\
\hline Stainless Steel (SS316) & 29.4 & 56.33 & 25.7 & 3.14 & 2.75 & 2.78 \\
\hline Titanium (Grade 5) & 22.15 & 46.66 & 21.5 & 2.86 & 2.78 & 2.77 \\
\hline
\end{tabular}

*, Physical fractional weighted atomic and mass number; **, Effective atomic number calculated from atomic and electronic cross-section

resulting in Compton scattering which depends on electron density. A slight increase in effective atomic number was observed beyond $1 \mathrm{MeV}$ due to pair production which depends on $\mathrm{Z}^{2}$ and $\mathrm{E}$ and it becomes constant beyond $20 \mathrm{MeV}$.

\section{Effective Electron density (Nel)}

Electron densities calculated by theoretical formula 9 and by experimental method with formulae 10 and 11 for stainless steel (SS316) and titanium (Grade5) are given in Table 7.

The electron density calculated from experimental value by using $Z_{\text {eff }}$ and electronic cross-section shows lower values than the theoretical values. Although the mass attenuation, total atomic and electronic cross-section varies with respect to change with energy, the electron density remains constant for all energy ranges of megavoltage $\mathrm{x}$-ray beams.

\section{Photon Mean Free Path ( $m f p)$}

The photon mean free path ( $\mathrm{mfp})$ is calculated experimentally $( \pm 0.1 \%)$ from linear attenuation coefficient using narrow beam geometry. It shows that path length increases with an increase in energy at central axis and off-axis as well. But the values at off-axis are less than that of the central axis because of the decrease in mean energy. A higher deviation was observed in flattened x-ray beams $(6 \mathrm{FF}, 10 \mathrm{FF}$ and $15 \mathrm{FF})$ with a maximum variation for $10 \mathrm{FF}$ at $10.3 \%$. The observed variation in $\mathrm{mfp}$ was less in unflattened $\mathrm{x}$-ray beam in comparison to flattened beam with a maximum variation with only $4.5 \%$ for $10 \mathrm{UF}$. The mfp is higher in titanium (Grade5) because its density is less than that of stainless steel (SS316). The mean free path here is not for mono-energetic $\mathrm{x}$-ray beam; it is for spectrum of energy with max energy as mentioned in Table 8.

\section{Discussion}

In this work, the MAC $(\mu / \rho)$ compound calculated for flattened and unflattened $\mathrm{x}$-ray beam using stainless steel (SS316) and titanium (Grade 5) shows a decrease in MAC with the increase in energy because of penetration ability. It increases for unflattened $\mathrm{x}$-ray beam owing to beam softening (relatively higher number of low energy photons) compared to respective flattened $\mathrm{x}$-ray beam energies. An incorporated mean energy from MAC shows that there is a change in the mean energy from flattened beam to unflattened x-ray beam; at off-axis, it decreases because of spectral variation. The total atomic and electronic cross-section calculated from MAC by theoretical methods show that the cross-section decreases with increase in energy and is relatively higher for flattened beam than unflattened x-ray beams. At off-axis $15 \mathrm{~cm}$ from central axis, the value decreases with respect to central axis due to the decrease in the mean energy. It is observed that an effective atomic number $\left(\mathrm{Z}_{\text {eff }}\right)$ remains constant irrespective of spectral changes between flattened or unflattened $\mathrm{x}$-ray beams in Compton region. However, in the photo electric effect energy range, the $Z_{\text {eff }}$ value drastically changes with respect to energy until Compton Effect becomes dominant. Electron density remains

Table 8. Mean Free Path (mfp) for Stainless Steel (SS316) and Titanium (Grade 5) for Flattened and Un-flattened X-ray Beam for Central and Off-Axis

\begin{tabular}{lcccccc}
\hline & \multicolumn{3}{c}{ Mean Free Path (mfp) in cm } & \multicolumn{3}{c}{ Titanium (Grade 5) } \\
Energy (MV) & CAX (0cm) & OAD (15cm) & \% Diff & CAX (0cm) & OAD (15cm) & $\%$ Diff \\
\hline $6 \mathrm{FF}$ & 2.88 & 2.62 & 9.00 & 5.12 & 4.71 & 8.00 \\
$10 \mathrm{FF}$ & 3.49 & 3.13 & 10.30 & 6.55 & 5.88 & 10.20 \\
$15 \mathrm{FF}$ & 3.81 & 3.45 & 9.40 & 7.17 & 6.51 & 9.20 \\
$6 \mathrm{UF}$ & 2.5 & 2.43 & 2.80 & 4.42 & 4.3 & 2.70 \\
$10 \mathrm{UF}$ & 3.08 & 2.94 & 4.50 & 5.64 & 5.46 & 3.20 \\
\hline
\end{tabular}

2490 Asian Pacific Journal of Cancer Prevention, Vol 20 
constant throughout Compton energy region for all energies studied here. The mfp is high for titanium (Grade 5) than stainless steel (SS316). The flattened x-ray beam shows relatively larger variation of $\mathrm{mfp}$ at off-axis because of drastic change in mean energy compared to unflattened $\mathrm{x}$-ray beams. With this fact, while treating the patient in the presence of high $\mathrm{Z}$ material in the treatment vicinity with the help of flattened and unflattened megavoltage x-ray beams, the interaction parameters can be well understood while change in energy spectrum (mean energy) that affects the above studied parameters.

\section{Acknowledgments}

We thank Mr. Sunder from Kalyani Radiotherapy Specialty India (P) Ltd for helping us to design Perspex phantom and high $\mathrm{Z}$ materials for this project. We sincerely thank our Managing Trustee Dr. B. S. Srinath for his motivation in doing this work. This study is not funded by any organization.

\section{Statement of Conflict of Interest}

There is no conflict of Interest.

\section{References}

Berger MJ, Hubbell JH (1987). XCOM: Photon cross section data base, NBSIR. (http://physics.nist.gov/xcom).

Cashmore J (2008). The characterization of Un-flattened photon beams from a $6 \mathrm{MV}$ linear accelerator. Phys Med Biol, 53, 1933-46.

Chester R, Rodica A, Inra JD, et al (2003). Dosimetric consideration for patient with HIP prostheses undergoing pelvic irradiation. Report of the AAPM Radiation Therapy Committee Task Group 63. Med Phys, 30, 1162-82.

Dalaryd M, Kragl G, et al (2010). A Monte Carlo study of a flattening filter-free linear accelerator verified with measurements. Phys Med Biol, 55, 7333-44.

Graham D, Paul C, Martin V (2012). Principle and Application of Radiological Physics, 6th Edition: Churchill Livingston Elsevier.

Goerg D, Knoos T, McClean B (2011). Current status and future prospective of flattening filter free photon beams. Med Phys, 38, 1280-93.

Hine GJ (1952). Physics Review. 82, 385.

Kaginelli SB, Rajeshwari T, Sharanabasappa, BR, et al (2009). Effective atomic number and electron density of dosimetric material. J Med Phys, 34, 176-9.

Kargl G, Aetterstedt AF, Knausl SB, et al (2009). Dosimetric characteristics of 6 and $10 \mathrm{MV}$ un-flattened photon beams. Radiotherapy Oncol, 40, 141-6.

Kerur BR, Thontadarya SR, Hanumaiah B (1993). Measurement of $\mathrm{x}$-ray mass attenuation coefficient using mixed radiation emitters. Indian J Phys, 67, A-23.

Khan FM (1994). The physics of Radiation Therapy. Baltimore: Williamson and Wilkins.

Kiran Kumar T, Vankata Reddy K (1997). Effective atomic number for materials of dosimetric interest. Radiat Phys Chem, 50, 545-53.

Koc N, Ozyol H (2000). Z-dependence of partial and total photon interactions in some biological samples. Radiat Phys Chem, 59, 339-45.

Manohara SR, Hanagodimath SM, Gerward L (2008). Studies on effective atomic number, electron density and kerma for some fatty acids and carbohydrates. Phys Med Biol, 53, 377-86.

Nowotny R (1998). XMuDat: photon attenuation data on PC, Tech. Rep. IAEA-NDS-195, International Atomic Energy Agency, Vienna, Austria, https://www.nds.iaea.org/ publications/iaea-nds/iaea-nds-0915.htm.

Papinakolaou N, Battista JJ, Boyar Al, et al (2004). Tissue Inhomogeneity correction for mega voltage photon beams: Report of Task Group No.65of the Radiation therapy committee. AAPM Report No. 85. Madisonm WI: Medical Physics Publishing.

Shivaramu H (2002). Effective atomic numbers for photon energy absorption and photon attenuation of tissues from human organs. Med Dosim, 27, 1-9.

Taylor ML, Smith RL, Dossing E, et al (2012). Robust calculation of effective atomic numbers: the auto $\mathrm{Z}_{\text {eff }}$ Software. Med Phys, 39, 1769-78.

TRS No. 398 (2000). Absorbed dose determination in external Beam radiotherapy: An international code of practice for dosimetry on standards of absorbed dose to water. Vienna: IAEA.

Weisner ME, Holden N, Coplen TB, et al (2013). Atomic Weights of the elements 2011 (IUPAC technical report). Pure Appl Chem, 85, 1047-78.

Zefkili S, Kappas C, Rosenwald JC (1994). On-axis and off-axis primary dose components in high energy photon beams. Med Phys, 21, 799-808.

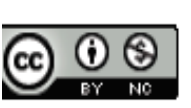

This work is licensed under a Creative Commons AttributionNon Commercial 4.0 International License. 